\title{
Spatial Point Analysis of Ion Track Patterns using Common Polymer Films by Atomic Forced Microscopy
}

\author{
Masaaki Omichi ${ }^{1,2, *}$, Wookjin $\mathrm{Choi}^{1}$, Satoshi Tsukuda ${ }^{3}$, Masaki Sugimoto ${ }^{4}$, and Shu Seki ${ }^{1{ }^{1 *}}$ \\ ${ }^{I}$ Department of Applied Chemistry, Graduate School of Engineering, \\ Osaka University, 2-1 Yamadaoka, Suita, Osaka 565-0871, Japan. \\ ${ }^{2}$ Center for Collaborative Research, Anan National College of Technology, \\ 265 Aoki Minobayashi, Anan, Tokushima 774-0017, Japan \\ ${ }^{3}$ Institute of Multidisciplinary Research for Advanced Materials, \\ Tohoku University, 2-1-1, Katahira, Sendai, Miyagi 980-8577, Japan \\ ${ }^{4}$ Japan Atomic Energy Agency, Takasaki Advanced Radiation Research Institute, \\ 1233 Watanuki-machi, Takasaki, Gunma 370-1292, Japan \\ seki@chem.eng.osaka-u.ac.jp
}

keywords: Spatial point analysis, Nearest neighbor method, Charged particle, Ion track

\section{Introduction}

Charged particle irradiation has attracted growing interest in cancer radiation therapy, because charged particle irradiation is expected ultimately to attack one tumor cell without damage to peripheral cells [1]. One of the essential matters to be solved for medical use of the charged particle is the spatial pattern analysis which an ion track pattern is uniformly distributed, randomly distributed, or clustered, though the charged ion particles are generally considered to be randomly irradiated.

We have developed a sensitive method for visualization of single ion track at ultra-high resolution using common polymer film, polyacrylic acid (PAA) $-N, N^{\prime}$-methylene bisacrylamide (MBAAm) blend film, by atomic forced microscopy (AFM). The visualization method is expected to be successfully applied to spatial pattern analysis of ion track with ultra-high spatial resolution [2].

Nearest neighbor analysis is widely used in various fields, such as population ecology [3], epidemiology [4], and bio-imaging [5]. In this analysis, spatial pattern is determined easily to measure the nearest neighbor distance of ion track. Here, we report spatial point analysis of ion track pattern by the combination of ion track visualization with PAA-MBAAm films and the nearest neighbor analysis, demonstrating fairly clear random special distribution of ion tracks.

\section{Experimental}

PAA-MBAAm blend films on a silicon substrate $\left(1.0 \times 1.0 \mathrm{~cm}^{2}\right)$ were prepared by spin-casting with a methanol solution of $3 \mathrm{wt} \%$ PAA with MBAAm (PAA/MBAAm $=75 / 25$ ). The thickness of the films was adjusted to 500 $\mathrm{nm}$. The films were irradiated in a vacuum chamber $\left(<10^{-4} \mathrm{~Pa}\right)$ with $490 \mathrm{MeV}{ }^{192} \mathrm{Os}^{30+}$ ion beams from the cyclotron accelerator at Takasaki Advanced Radiation Research Institute, Japan Atomic Energy Agency. The expected fluence of the incident ions varied from $5.0 \times 10^{7}$ to $2.5 \times$ $10^{8}$ ions $\mathrm{cm}^{-2}$. After irradiation, the films were reserved in the desiccator with dry silica gel under reduced pressure at $25^{\circ} \mathrm{C}$. The reserved films were moved into the desiccator saturated with water vapor at $25^{\circ} \mathrm{C}$. After standing for 3 $\mathrm{min}$, the film surfaces were observed by AFM. The AFM images were obtained with a Naio AFM (NanoSurf, Switzerland) operated in tapping mode in air at $25^{\circ} \mathrm{C}$. Scan size was over 6 $\times 6 \mu \mathrm{m}$. For the fluence of $5.0 \times 10^{7}$ ions $\mathrm{cm}^{-2}, 16$ points were randomly scanned in the film. For the fluence of $1.0 \times 10^{8}$ and $2.5 \times 10^{8}$ ions $\mathrm{cm}^{-2}, 8$ points were randomly scanned in the film.

The spatial point analysis of ion track pattern was carried out by using nearest neighbor analysis [3]. To minimize the edge effect, nearest neighbor analysis was performed in the smaller

Received April 5, 2014

Accepted May 15, 2014 
square $(5 \times 5 \mu \mathrm{m})[6]$. The observed mean nearest neighbor distance (NND) of ion tracks, represented by $r_{\mathrm{A}}$, is equal to

$$
\bar{r}_{\mathrm{A}}=\frac{\sum r}{N}
$$

where $N$ is the total number of ion tracks in analysis areas and $r$ is the distance from a given individual to its nearest neighbor. The expected mean NND of ion tracks in completely random distribution, represented by $\bar{r}_{\mathrm{E}}$, is equal to

$$
\bar{r}_{\mathrm{E}}=\frac{1}{2(N / A)^{1 / 2}}
$$

where $A$ is the size of analysis area. The standard error of expected mean NND, represented by $\sigma_{\overline{\bar{t}_{\mathrm{E}}}}$, is equal to

$$
\sigma_{\bar{\tau}_{\mathrm{E}}}=\left(\frac{4-\pi}{4 \pi N^{2}} A\right)^{1 / 2}=\frac{0.26136 A^{1 / 2}}{N}
$$

The standard variate of the normal curve, represented by $c$, is equal to

$$
c=\frac{\bar{r}_{\mathrm{A}}-\bar{r}_{\mathrm{E}}}{\sigma_{\bar{r}_{\mathrm{E}}}}
$$

The mesure of the degree to which observed distribution approachs or departs from random expectation with respect to NND, represented by $R$, is equal to

$$
R=\frac{\bar{r}_{\mathrm{A}}}{\bar{r}_{\mathrm{E}}}
$$

In a typical random pattern, the value of $R$ is 1 . The value of $R<1$ indicates clusterd, while the value $R>1$ indicating regular spacing.

\section{Results and discussion}

Figure 1a shows the AFM image of the 4 different areas in the PAA-MBAAm blend films irradiated with $490 \mathrm{MeV}{ }^{192} \mathrm{Os}^{30+}$ ion beams at expected fluence of $1.0 \times 10^{8}$ ions $\mathrm{cm}^{-2}$ and exposed to saturated water vapor. The irradiated spatial points of the film swelled significantly, giving corresponding peaks of the bright spots in the Figure. Ion tracks are observed to be inhomogeneous patterns in a certain area. To analyse spatial pattern, each NND of ion tracks in analysis areas $\left(200 \mu^{2}\right)$ was measured. Figure $1 \mathrm{~b}$ shows the NND histogram at expected fluence of $1.0 \times 10^{8}$ ions $\mathrm{cm}^{-2}\left(=1.0\right.$ ions $\left.\mu \mathrm{m}^{-2}\right)$. In analysis areas, 140 ion tracks were counted and the observed fluence is 0.70 ions $\mu \mathrm{m}^{-2}$, which is slightly lower than the fluence incident charged particles estimated from transient current monitored with Faraday-cup measurement. (1.0 ions $\mu \mathrm{m}^{-2}$ ). For all 140 ion tracks in $200 \mu \mathrm{m}^{2}$, the observed mean NND is $611 \mathrm{~nm}$ (Equation (1)). If these charged ion particles were randomly irradiated, the expected NND is $598 \mathrm{~nm}$ with a standard error of $26 \mathrm{~nm}$ (Equations (2) and (3)). The value of standard normal variate is a quite small (0.51), and the value of $\mathrm{R}$ (1.02) was close to 1 (Equations (4) and (5)). There are little difference between observed ion track distribution and theoretical random pattern, indicating that the ion track distribution is a random pattern.

(a)

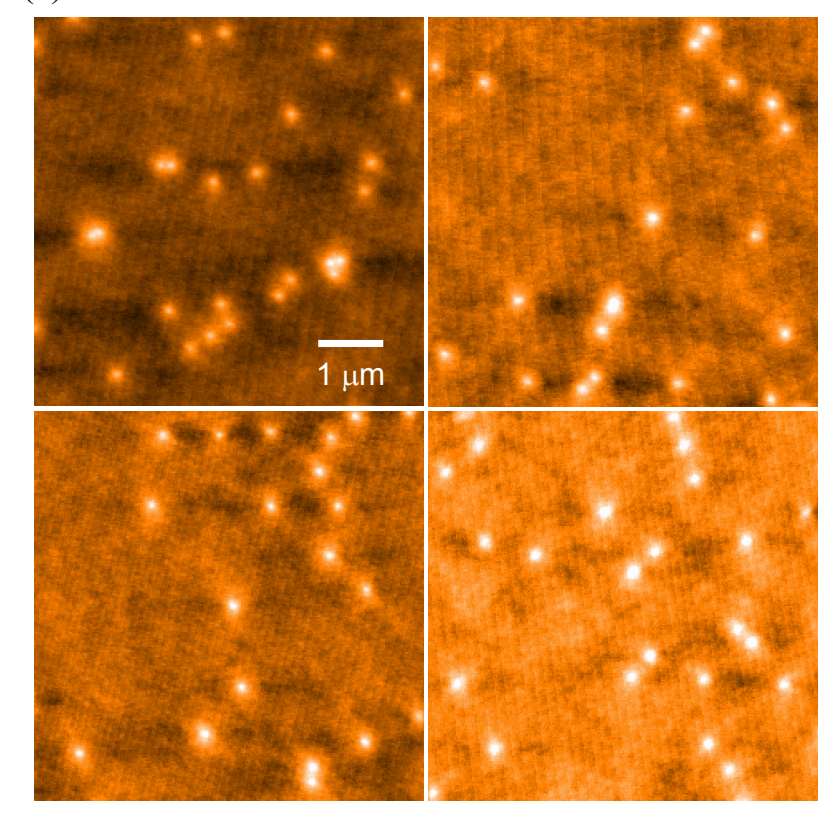

(b)

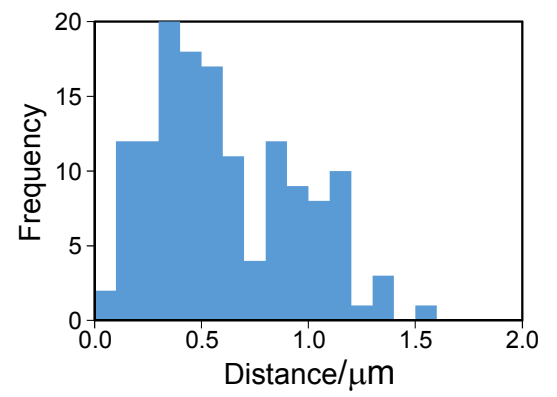

Fig. 1. (a) AFM images of the 4 different areas in the PAA-MBAAm film with $490 \mathrm{MeV}{ }^{192} \mathrm{Os}^{30+}$ ion beam at expected fluence of $1.0 \times 10^{8}$ ions $\mathrm{cm}^{-2}$. (b) NND histogram $(n=140)$.

To examine the effect of the fluence on the spatial pattern of ion track, the nearest neighbor method was also applied to the expected fluence of 0.50 and 2.5 ions $\mu \mathrm{m}^{-2}$. Ion tracks were increased with increasing the fluence, as shown in Fig. 2a. The distribution of NND significantly changed depending on the fluence (Fig. 2b). The each observed fluence is 0.38 and 1.5 ions $\mu \mathrm{m}^{-2}$, respectively. The observed number of ion tracks 
in a unit area is directly proportional to the fluence of incident charged particles estimated from transient current monitored with Faraday-cup measurement. Table 1 summarizes nearest neighbor analysis of ion track pattern. These results indicate that the ion track distribution is well interpreted by a random pattern model in the area, which is also independent of fluence.

To evaluate the details of spatial pattern of ion track, the cumulative probability of point event distances to the nearest ion track were calculated from the distribution of NND (Fig. 3).

(a)

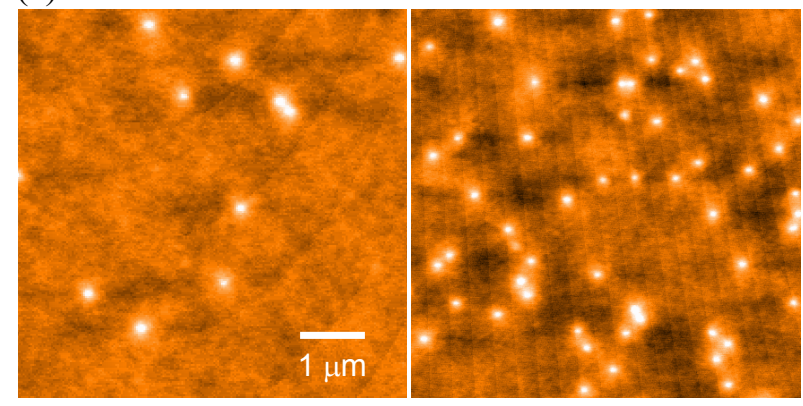

(b)
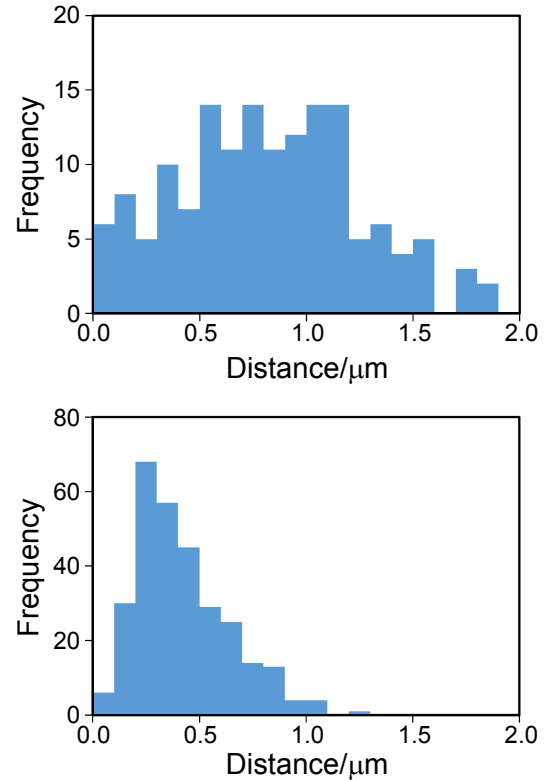

Fig. 2. (a) AFM images of the PAA-MBAAm films with $490 \mathrm{MeV}{ }^{192} \mathrm{Os}^{30+}$ ion beam at expected fluence of $5.0 \times 10^{7}$ (left) and $2.5 \times 10^{8}$ ions $\mathrm{cm}^{-2}$ (right). (b) NND histogram at expected fluence of $5.0 \times 10^{7}(n=$ 151 , upper) and $2.5 \times 10^{8}$ ions $\mathrm{cm}^{-2}(n=296$, lower $)$.

In a theoretical random pattern, the cumulative probability, $F(r)$, is

$$
F(r)=1-e^{-\pi N r^{2} / A}
$$

In the all fluence, the observed cumulative probabilities agree mostly with the theoretical
Table 1. Comparison of the various fluence in the application of the nearest neighbor method.

\begin{tabular}{lccc}
\hline & Run 1 & Run 2 & Run 3 \\
\hline Expected fluence (ions $\left./ \mu \mathrm{m}^{2}\right)$ & 0.50 & 1.0 & 2.5 \\
Scan area $\left(\mu \mathrm{m}^{2}\right)$ & 400 & 200 & 200 \\
Number of ion tracks & 151 & 140 & 296 \\
Observed fluence $\left(\right.$ ions $\left./ \mu^{2}\right)$ & 0.38 & 0.70 & 1.5 \\
Observed mean NND $(\mathrm{nm})$ & 812 & 611 & 422 \\
Expected mean NND $(\mathrm{nm})$ & 816 & 598 & 411 \\
$\sigma_{\bar{T}_{\mathrm{E}}}(\mathrm{nm})$ & 35 & 26 & 12 \\
$c$ & -0.05 & 0.51 & 0.88 \\
$R$ & 1.00 & 1.02 & 1.03 \\
\hline
\end{tabular}

(a)

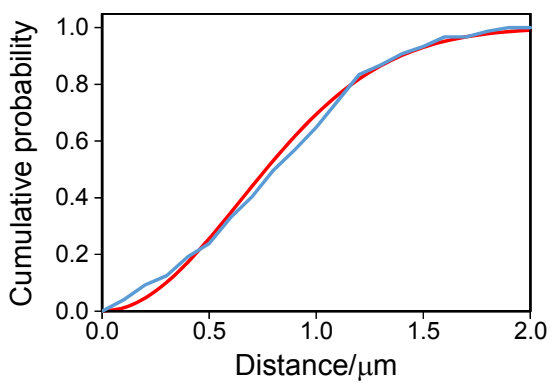

(b)

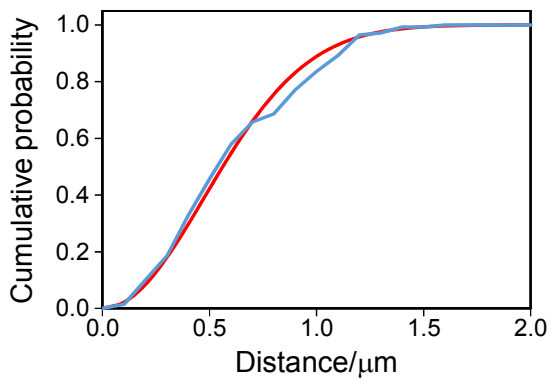

(c)

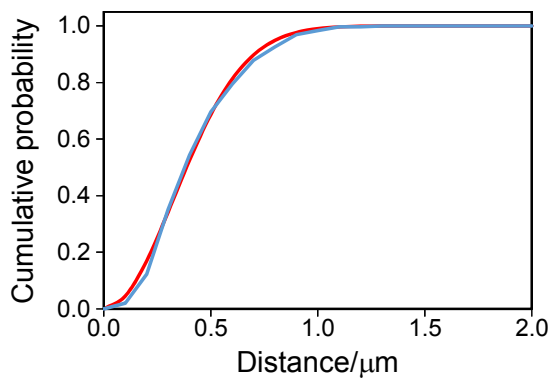

Fig. 3. Cumulative probability of point event distances to the nearest ion track at observed fluence of (a) 0.38 , (b) 0.70 , and (c) 1.5 ions $\mu m^{-2}$. (blue) Observed cumulative probability. (red)

Theoretical cumulative probability with completely random pattern.

cumulative probability. Random patterns, again, are reproducible to the distribution of ion tracks, 
and the charged ion particle rarely interferes with another charged ion particles at low fluence. This is the first report that nearest neighbor method was also applied to track pattern. This analysis will be contributed to not only fundamental study but also applied study such as cancer therapy.

\section{Acknowledgements}

This work was supported by the JSPS Funding Program for Next-Generation World-Leading Research (NEXT Program), a Grant-in-Aid for Scientific Research (S) from MEXT Japan (No. 22226017), Grant-in-Aid for Young Scientists (B) (No. 25810129), and a Sekisui Chemical Grant Program for Research. This work was performed under the Shared Use Program of JAEA Facilities.

\section{References}

1. R. R. Wilson, Radiology, 47 (1946) 487; G. Kraft,
Prog. Part. Nucl. Phys., 45 (2000) S473; S. Rieken, D. Habermehl, A. Nikoghosyan, A. Jensen, T. Haberer, O. Jäkel, M. W. Münter, T. Welzel, J. Debus, and S. E. Combs, Int. J. Radiat. Oncol. Biol. Phys., 81 (2010) e 793.

2. M. Omichi, K Takano, T. Satoh, T. Kamiya, Y. Ishii, T. Ohkubo, M. Koka, W. Kada, M. Sugimoto, H. Nishikawa, and S. Seki, J. Nanosci. Nanotechnol., 12 (2012) 7401.

3. P. J. Clark, and F. C. Evans, Ecology, 35 (1954) 23.

4. S. Selvin, K. E. Ragland, Y. L. Chien, and P. A. Buffler, Int. J. Hyg. Environ. Health, 207 (2004), 555.

5. B. I. de Bakker, F. de Lange, A. Cambi, J. P. Korterik, E. M. van Dijk, N. F. van Hulst, C. G. Figdor and M. F. Garcia-Parajo, Chem Phys. Chem., 8 (2007) 1473.

6. D. F. Sinclair, Ecology, 66 (1985) 1084. 